\title{
Identificação de um motor de indução trifásico aplicado para posicionamento utilizando modelo de Hammerstein
}

\author{
Identification of a three-phase induction motor used for positioning \\ using Hammerstein model
}

Lucas Rabelo Rebouças lucasrabeloreboucas@gmail. com Universidade Federal do Ceará

\section{Antônio Barbosa de Souza Júnior \\ barbosa@dee.ufc.br Universidade Federal do Ceará}

\section{Tobias Rafael \\ Fernandes Neto \\ tobias@dee.ufc.br Universidade Federal do Ceará}

\section{Laurinda Lúcia} Nogueira dos Reis laurinda@dee.ufc.br Universidade Federal do Ceará

\author{
Luiz Henrique Silva \\ Colado Barreto \\ lbarreto@dee.ufc.br \\ Universidade Federal do \\ Ceará
}

\begin{abstract}
Resumo
Este trabalho apresenta a identificação de um motor de indução trifásico, do tipo gaiola de esquilo, utilizado como servoposicionador, empregando-se o modelo não linear de Hammerstein, o qual é composto por um bloco estático não linear seguido de um bloco linear dinâmico. A representação de um processo através de um modelo não linear visa melhorar o seu desempenho, pois consegue representar as dinâmicas existentes no processo. Utilizou-se um potenciômetro de baixo custo para a realimentação do sistema, o que provoca ruídos, fazendo com que a técnica não linear seja ainda mais importante, além de suportar variações nos parâmetros do sistema, inerentes ao processo. Ao final do trabalho, é realizada a validação do modelo estimado, comparando-o com os dados coletados experimentalmente e avaliando-se seu desempenho através de índices de validação.
\end{abstract}

Palavras-chave: Identificação não linear, Hammerstein, servo posicionamento, motor de indução trifásico.

\begin{abstract}
This paper presents the identification of a three-phase induction motor of squirrel cage used as positioner servo, using the nonlinear Hammerstein model, which comprises a nonlinear static block followed by a linear dynamic block. The representation of a process using a nonlinear model aims to improve its performance, because it can represent the existing dynamics in the process. We used a low cost potentiometer for the feedback system, which causes noise, causing nonlinear technique is even more important and supports variations in system parameters of the process. At the end of the paper is performed to validate model comparing it with data collected experimentally and evaluating performance through validation indices.
\end{abstract}

Keywords: Nonlinear identification, Hammerstein, servo positioning, induction motor.

\section{Introdução}

Com a tendência de processos industriais cada vez mais complexos, é de importância o conhecimento dos modelos matemáticos para auxiliar na análise, projeto e entendimento do funcionamento dos sistemas de controle sob investigação (COELHO \& COELHO, 2004). A identificação de sistemas é a área do conhecimento que aborda a obtenção do modelo desejado através de observações, a fim de se relacionar as variáveis de entrada e saída do sistema. Uma das características dessas técnicas é que pouco ou nenhum conhecimento prévio do sistema é necessário (AGUIRRE, 2007). O grande interesse do uso da identificação de sistemas decorre dos significativos avanços tecnológicos nas áreas de engenharia elétrica, ciência da computação, medicina, entre outras. 
A identificação de sistemas é a determinação do modelo matemático de um sistema representando os seus aspectos essenciais de forma adequada para uma utilização particular (diagnóstico, supervisão, otimização, controle). Dessa forma, propicia a obtenção de modelos que representam a dinâmica do sistema. Para fins de controle, não se pretende encontrar um modelo matemático exato, mas um modelo adequado para uma determinada aplicação (COELHO \& COELHO, 2004).

Dentre os diversos modelos para identificação de sistemas, existem os modelos lineares e não lineares. Este artigo utiliza o modelo não linear de Hammerstein para obter o modelo de um motor de indução trifásico (MIT), do tipo gaiola de esquilo, que é utilizado para posicionamento de seu eixo.

O modelo de Hammerstein tem por sua característica que a não linearidade estática precede o modelo dinâmico linear, conforme será mostrado. Visando aliar a capacidade de representação da não linearidade de um processo com a simplicidade de projeto, torna-se interessante a utilização de modelos de Hammerstein.

Diniz et al. (2010) destacam que estudos sobre acionamento de motores elétricos são fundamentais no que diz respeito à eficiência, performance dinâmica, flexibilidade de operação, fácil diagnóstico de falhas e comunicação com um computador central. Além disso, a literatura destaca duas estratégias clássicas de acionamento, sendo a tradicional com controle escalar e a mais recente, utilizando controle de campo orientado. Como a maioria das aplicações industriais utiliza motor de indução trifásico, escolheu-se o controle de campo orientado de forma que seu desempenho seja similar às máquinas de corrente contínua.

Geralmente, o modelo matemático de um motor de indução trifásico necessita construir um sistema de controle, o que significa que todos os parâmetros devem ser identificados previamente ou em tempo real. Especialmente alguns parâmetros têm que ser identificados on-line, pois a dinâmica do motor de indução pode mudar devido a parâmetros de perturbação, que é causada pela variação da temperatura ou condição da carga (HASEGAWA et al., 2008).

Este trabalho consiste em identificar o modelo do motor de indução trifásico aplicado para servo posição, utilizando técnicas de identificação de sistemas não lineares, tal qual o modelo de Hammerstein, a fim de se obter um modelo que represente mais fielmente a dinâmica do processo.

\section{Modelagem Dinâmica da Máquina de Indução Trifásica}

O diagrama de blocos de um sevo posicionador utilizando controle vetorial indireto e um motor de indução trifásico é mostrado na Figura 1.

Figura 1: Diagrama de blocos do servo posicionador.

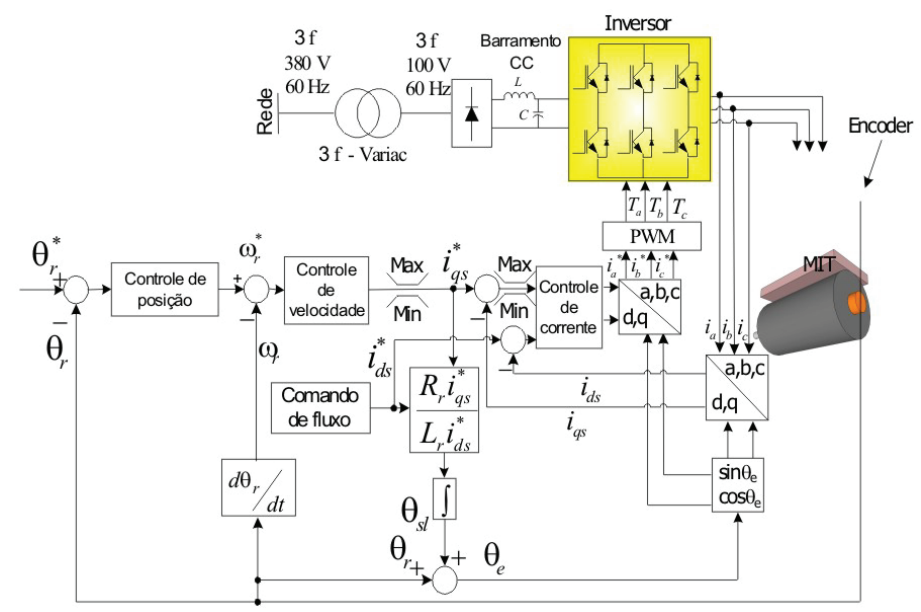

O acionador consiste principalmente de um servo motor de indução, um mecanicos de orientação de campo, um transformador de coordenadas ( $\mathrm{ABC}$ para dq0), uma malha de controle de velocidade interna e uma malha de controle de posição externa.

O equacionamento de um motor de indução com referência girante síncrona pode ser escrita da seguinte forma (BOSE, 1996): 
Sendo,

$$
x=\left[\begin{array}{c}
i_{d s} \\
i_{q s} \\
\lambda_{d r} \\
\lambda_{q r}
\end{array}\right] ; \boldsymbol{B}=\left[\begin{array}{cccc}
\frac{1}{\sigma L_{s}} & 0 & 0 & 0 \\
0 & \frac{1}{\sigma L_{s}} & 0 & 0 \\
0 & 0 & 0 & 0 \\
0 & 0 & 0 & 0
\end{array}\right] ; u=\left[\begin{array}{c}
v_{d s} \\
v_{q s} \\
0 \\
0
\end{array}\right] ; \boldsymbol{A}=\left[\begin{array}{cccc}
-\frac{R_{s}}{\sigma L_{s}}-\frac{R_{r}(1-\sigma)}{\sigma L_{r}} & \omega_{e} & \frac{L_{m} R_{r}}{\sigma L_{s} L_{r}^{2}} & \frac{P \omega_{r} L_{m}}{2 \sigma L_{s} L_{r}^{2}} \\
\omega_{e} & -\frac{R_{s}}{\sigma L_{s}}-\frac{R_{r}(1-\sigma)}{\sigma L_{r}} & \frac{-P \omega_{r} L_{m}}{2 \sigma L_{s} L_{r}^{2}} & \frac{L_{m} R_{r}}{\sigma L_{s} L_{r}^{2}} \\
\frac{L_{m} R_{r}}{L_{r}} & 0 & -\frac{R_{r}}{L_{r}} & \omega_{e}-\frac{P}{2} \omega_{r} \\
0 & \frac{L_{m} R_{r}}{L_{r}} & -\left(\omega_{e}-\frac{P}{2} \omega_{r}\right) & -\frac{R_{r}}{L_{r}}
\end{array}\right]
$$

A equação do torque eletromagnético é dada por:

$$
T_{e}=\frac{3 P}{4} \frac{L_{m}}{L_{r}}\left(i_{q s} \lambda_{d r}-i_{d s} \lambda_{q r}\right)
$$

E as demais variáveis:

$$
\begin{aligned}
& \sigma=1-\frac{L_{m}^{2}}{L_{s} L_{r}} \\
& \lambda_{q r}=L_{m} i_{q s}+L_{r} i_{d r} \\
& \lambda_{d r}=L_{m} i_{d s}+L_{r} i_{q r}
\end{aligned}
$$

Em que

$\mathrm{R}_{\mathrm{S}}$ : Resistência estatórica por fase;

$\mathrm{L}_{\mathrm{S}}$ : Indutância de magnetização estatórica por fase;

$\mathrm{R}_{\mathrm{r}}$ :Resistência rotórica por fase referenciada ao estator;

$\mathrm{L}_{\mathrm{r}}$ : Indutância rotórica por fase referenciada ao estator;

$\mathrm{L}_{\mathrm{m}}$ : Indutância de magnetização por fase;

P : Número de polos;

$\omega_{\mathrm{s}}$ : Velocidade elétrica angular;

$\omega_{\mathrm{s}}$ : Frequência de escorregamento angular;

$v_{\mathrm{ds}}$ : Tensão do estator no eixo direto;

$v_{\mathrm{qs}}$ : Tensão do estator no eixo em quadratura;

$i_{\text {ds }}$ : Corrente do estator no eixo direto;

$i_{\text {qs }}$ : Tensão do estator no eixo em quadratura.

Em um campo orientado ideal de um motor de indução, ocorre o desacoplamento entre os eixos direto e em quadratura e o fluxo rotórico de dispersão é alinhado ao eixo direto. Dessa forma, o fluxo de dispersão e sua derivada no eixo em quadratura é nula, ou seja:

$$
\omega_{s l}=\frac{L_{m} R_{r} i_{q s}^{*}}{L_{r} \lambda_{d r}}=\frac{R_{r} i_{q s}^{*}}{L_{r} i_{d s}^{*}}
$$

O fluxo rotórico de dispersão pode ser calculado através das terceira linha da matriz da Equação (1). Utilizando ainda a Equação (3), tem-se:

$$
\lambda_{q r}=0 \mathrm{e} \frac{d \lambda_{q r}}{d t}=0
$$


Fazendo a constante de tempo elétrica do sistema desprezível com relação à constante mecânica, a constante de tempo da Equação (4) torna-se próxima a zero e a corrente $i_{d s}$ se torna constante $\left(i_{d s}=i_{d s}^{*}\right)$ de modo a se ter um fluxo rotórico desejado constante. Desta forma, a Equação (4) se torna:

$$
\lambda_{d r}=\frac{L_{m} i_{d s}}{1+s \frac{L_{r}}{R_{r}}}
$$

Utilizando as Equações (3) e (5), a equação de conjugado (2) se torna:

$$
\lambda_{d r}=L_{m} i_{d s}^{*}
$$

Sendo $i_{q s}^{*}$ denota o comando de conjugado controlado pela corrente do estator no eixo em quadratura, sendo esta controlada por $\mathrm{G}_{S}(\mathrm{~s})$, mostrado na Figura

1. No método do campo orientado indireto, a frequência precisa ser calculada em coordenadas dq0. Utilizando a quarta linha da Equação (1) em conjunto com a Equação (3), a frequência de escorregamento pode ser estimada por:

$$
T_{e}=\frac{3 P}{4} \frac{L_{m}^{2}}{L_{r}} i_{d s}^{*} i_{q s}^{*}
$$

O conjugado gerado, a velocidade rotórica e a posição angular $\theta_{\Upsilon}$ são relacionados por:

$$
\omega_{r}=s \theta_{r}=\frac{1 / J}{s+B / J}\left[T_{e}(s)-T_{L}(s)\right]
$$

Sendo,

$B$ : Coeficiente de atrito viscoso;

$J$ : Constante do momento de inércia.

\section{Modelo de Hammerstein}

O modelo de Hammerstein é constituído de um elemento estático não linear, seguido por um sistema dinâmico linear, como mostrado na Figura 2.

Figura 2: Modelo de Hammerstein.

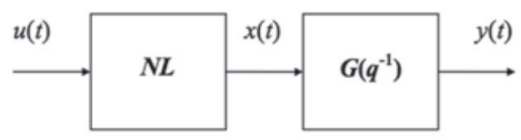

O bloco da não linearidade estática (NL) pode ser representado por um polinômio, pela equação da não linearidade ou através de modelos semiparamétricos (SANTOS, 2007).

\section{A. Representação da não linearidade por um polinômio}

Aproximando a não linearidade por uma expansão polinomial finita do tipo

$$
x(t)=\gamma_{1} u(t)+\gamma_{2} u^{2}(t)+\cdots+\gamma_{m} u^{m}(t)
$$

Em que:

$t:$ Instante de tempo;

$x(t)$ : Pseudo-saída, não mensurável, do bloco não linear;

$u(t)$ : Variável de entrada;

$\gamma_{i}(i=1, \ldots, m)$ : Coeficientes do polinômio;

$m$ : Grau de não linearidade do modelo. 
Normalmente, considera-se $\gamma_{1}=1$ transferindo o ganho estático para a parcela dinâmica linear, $\mathrm{G}\left(\mathrm{q}^{-1}\right)$, que pode ser representada por qualquer um dos modelos lineares.

O modelo de Hammerstein pode ser apresentado, ainda, como um caso particular do modelo de Volterra, tornando-se na forma paramétrica:

$$
A\left(q^{-1}\right)=b_{0}+B_{1}\left(q^{-1}\right) u(t-d)+\sum_{j=0}^{n u} b_{2} u^{2}(t-d-j)+\sum_{j=0}^{n u} b_{3} u^{3}(t-d-j)+\cdots+\varepsilon(t)
$$

Que pode ser reescrita como

$$
A\left(q^{-1}\right) y(t)=B\left(q^{-1}\right) \sum_{i=1}^{m} \gamma_{1} u^{i}(t-d)+\varepsilon(t)
$$

Ou, ainda, substituindo a Equação (12),

$$
A\left(q^{-1}\right) y(t)=B\left(q^{-1}\right) x(t)+\varepsilon(t)
$$

Que apresenta a relação linear entre a saída y(t) e a pseudo-saída x(t).

\section{B. Representação pela equação da não linearidade}

Este caso é aplicado quando a não linearidade envolvida apresenta uma estrutura conhecida como saturação, zonamorta, histerese ou relé, conforme a Figura 3.

Figura 3: Tipos de não linearidades.

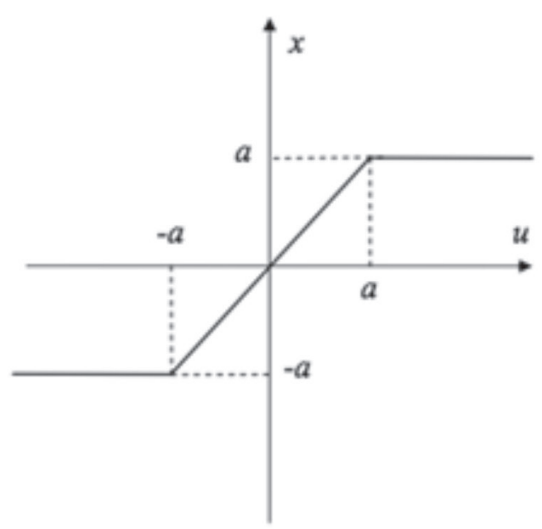

(a) Saturação

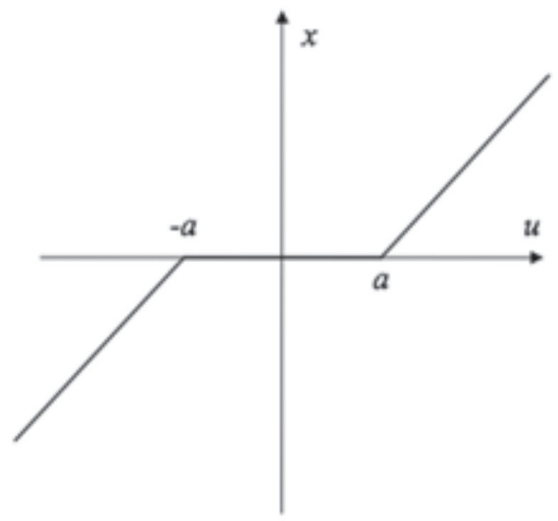

(c) Zona-Morta

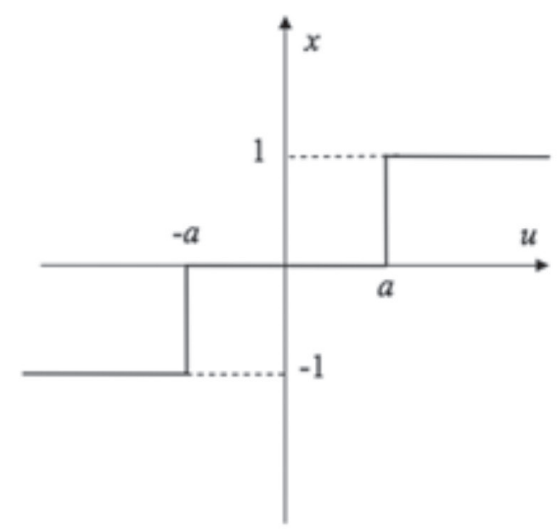

(b) Relé

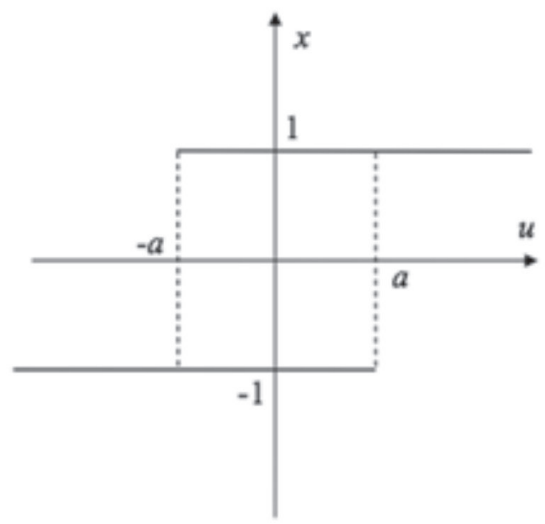

(d) Histerese 
As equações para cada tipo de não linearidade apresentada são:

Saturação:

$$
x(t)=\frac{1+\operatorname{sgn}(a-|u(t)|)}{2} u(t)+\frac{1+\operatorname{sgn}(|u(t)|-a)}{2} \operatorname{arggn}(u(t))
$$

Zona-morta:

$$
x(t)=u(t)-a \cdot \operatorname{sgn}(u(t))-\frac{1+\operatorname{sgn}(a-|u(t)|)}{2}[u(t)-a \cdot \operatorname{sgn}(u(t))]
$$

Histerese:

$$
x(t)=\frac{\operatorname{sgn}(u(t)-a)+\operatorname{sgn}(u(t)+a)}{2}
$$

Relé:

$$
x(t)= \begin{cases}u(t)-a, & u(t)>u(t-1) \\ u(t)+a, & u(t)<u(t-1) \\ x(t-1), & u(t)=u(t-1)\end{cases}
$$

\section{Representação da não linearidade por um modelo semiparamétrico}

Neste caso, os modelos são formados por números que correspondem às ponderações de uma rede neural artificial ou ao grau de pertinência num conjunto difuso.

Modelos Rede Neural Artificial - Esses modelos têm a capacidade de aprender o comportamento entrada-saída do sistema. Uma rede neural consiste de vários elementos computacionais simples, denominados nós, arranjados em camadas e operando em paralelo (Figura 4).

Figura 4: Estrutura de um modelo Hammerstein Neural.

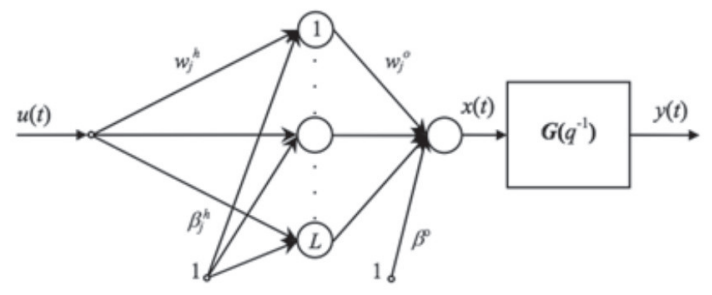

Modelos Nebulosos - Esses modelos combinam informação numérica e linguística (do tipo pequeno, médio, grande etc.), possibilitando a aplicação do conhecimento prévio das características do processo, mesmo que este esteja incompleto ou com incertezas (Figura 5).

Figura 5: Estrutura de um modelo Hammerstein Nebuloso.

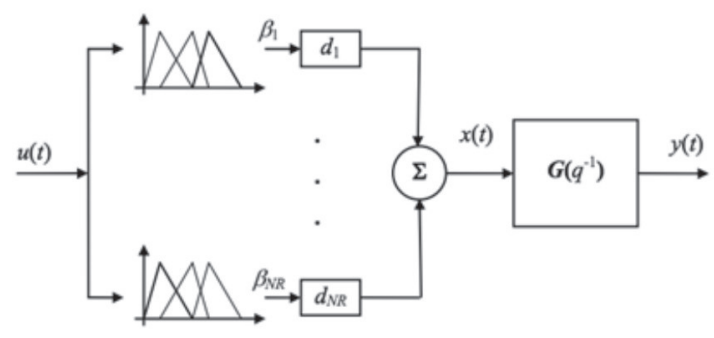

Fonte: Santos (2007).

A popularidade do modelo de Hammerstein deve-se ao fato de maior simplicidade em relação às representações de Volterra e Bilinear, aliada a uma capacidade de representação da não linearidade da maioria dos processos práticos, sendo capaz de representar processos com atuadores não lineares e ganhos variantes (SANTOS, 2007). 


\section{Resultados Experimentais}

Para implementação do sistema, foi construída uma bancada de desenvolvimento (Figura 6).

Figura 6: Bancada de desenvolvimento.

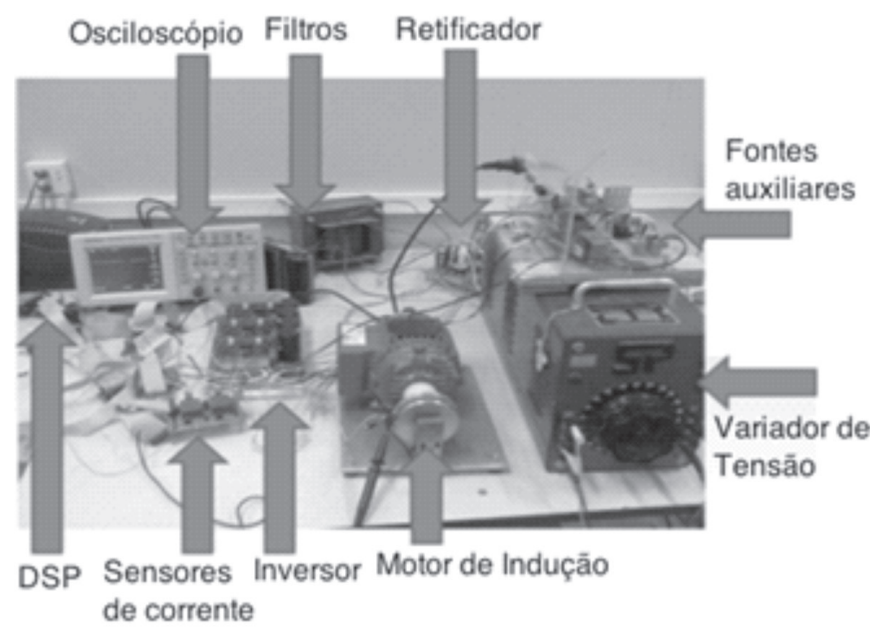

O motor utilizado foi uma máquina de potência fracionada de indução trifásica, do tipo gaiola de esquilo, conectada em delta com as seguintes características principais:

Tabela 1: Parâmetros do motor.

\begin{tabular}{lc}
\hline Parâmetro & Valor \\
\hline Potência nominal & $0,25 \mathrm{HP}$ \\
Tensão nominal & $220 \mathrm{~V}$ \\
Corrente nominal & $1,44 \mathrm{~A}$ \\
Número de polos & 4 \\
Frequência & $60 \mathrm{~Hz}$ \\
\hline
\end{tabular}

A realimentação do sistema é feita por um potenciômetro de baixo custo conectado ao eixo do motor. Dessa forma, apresenta uma realimentação ruidosa, como pode ser visto na Figura 8. Por isso a importância da utilização de uma técnica não linear para modelagem, pois, além dos ruídos apresentados, essa técnica suporta as possíveis variações dos parâmetros da máquina de indução, tal como a resistência rotórica, que varia com a temperatura e é um parâmetro essencial para o correto funcionamento do controle de campo orientado.

O proceso a ser modelado apresenta uma não linearidade do tipo zona-morta na entrada (Figura 7).

Figura 7: Processo com não linearidade do tipo zona-morta.

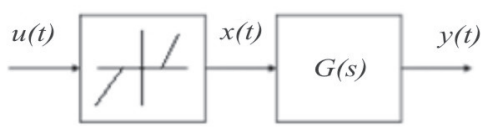

O comportamento da posição do eixo do motor é apresentado na Figura 8. Utilizando a técnica não linear de Hammerstein, o modelo para a parte linear dinâmica do processo é representado pela função de transferência a seguir:

$$
G(z)=\frac{0.5959 z^{-1}-0.5947 z^{-2}}{1-1.003 z^{-1}+0.09753 z^{-2}-0.09273 z^{-3}}
$$


Lucas Rabelo Rebouças, Antônio Barbosa de Souza Júnior, Tobias Rafael Fernandes Neto, Laurinda Lúcia Nogueira dos Reis, Luiz Henrique Silva Colado Barreto

Sendo a não linearidade do tipo zona-morta, representada pela Equação (17).

Para realizar a identificação do processo, foi utilizado um tempo de amostragem de 0,5 segundo. Ao longo da simulação, foi aplicado um sinal do tipo degrau, sendo sua itensidade aumentada no decorrer do experimento, no valor de um radiano, conforme Figura 8.

Figura 8: Entrada e saída para identificação.
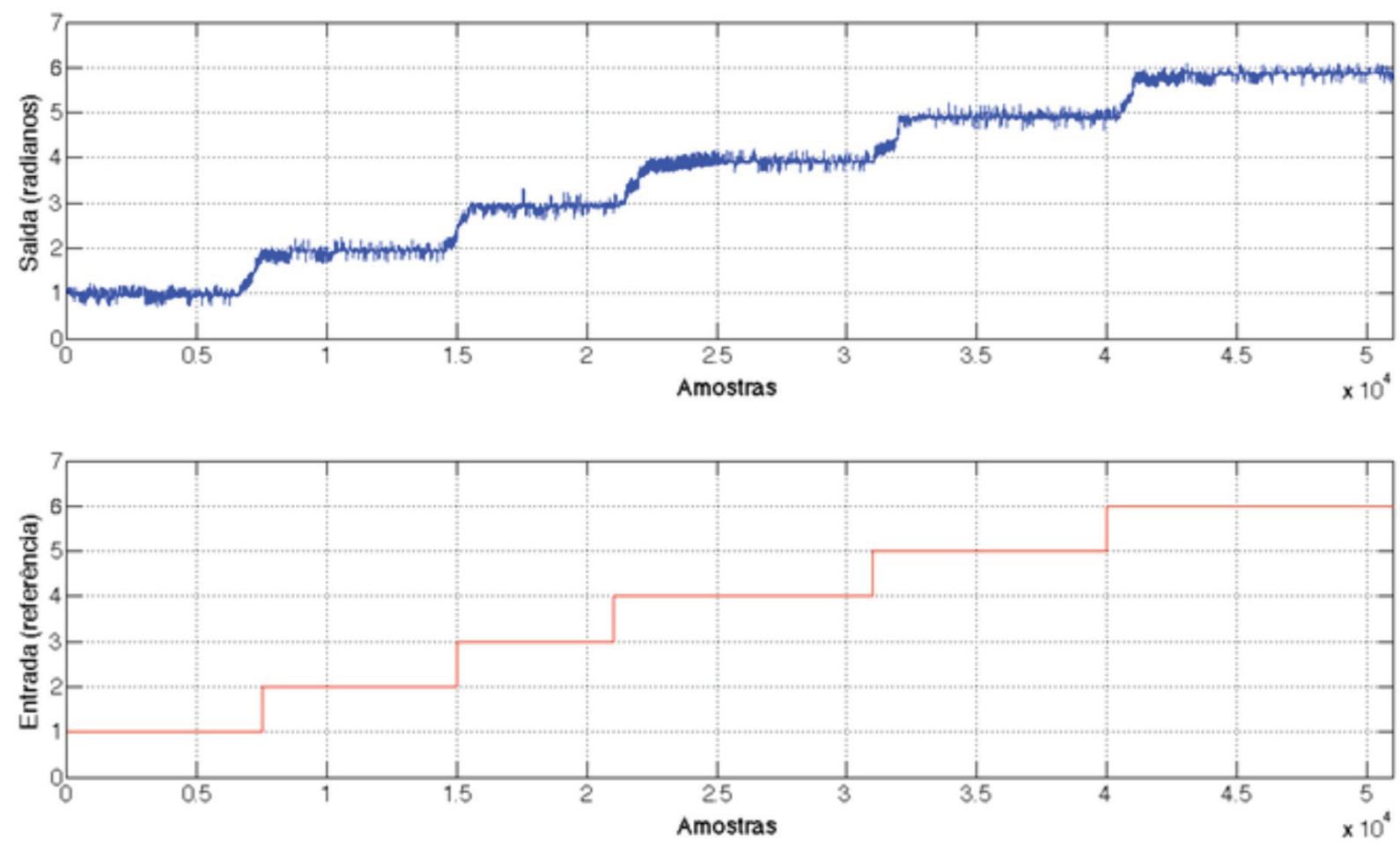

Utilizando-se os degraus de 1 até 3 radianos para identificação do modelo, obtém-se o resultado da Figura 9.

Figura 9: Comparação saída real x saída estimada.

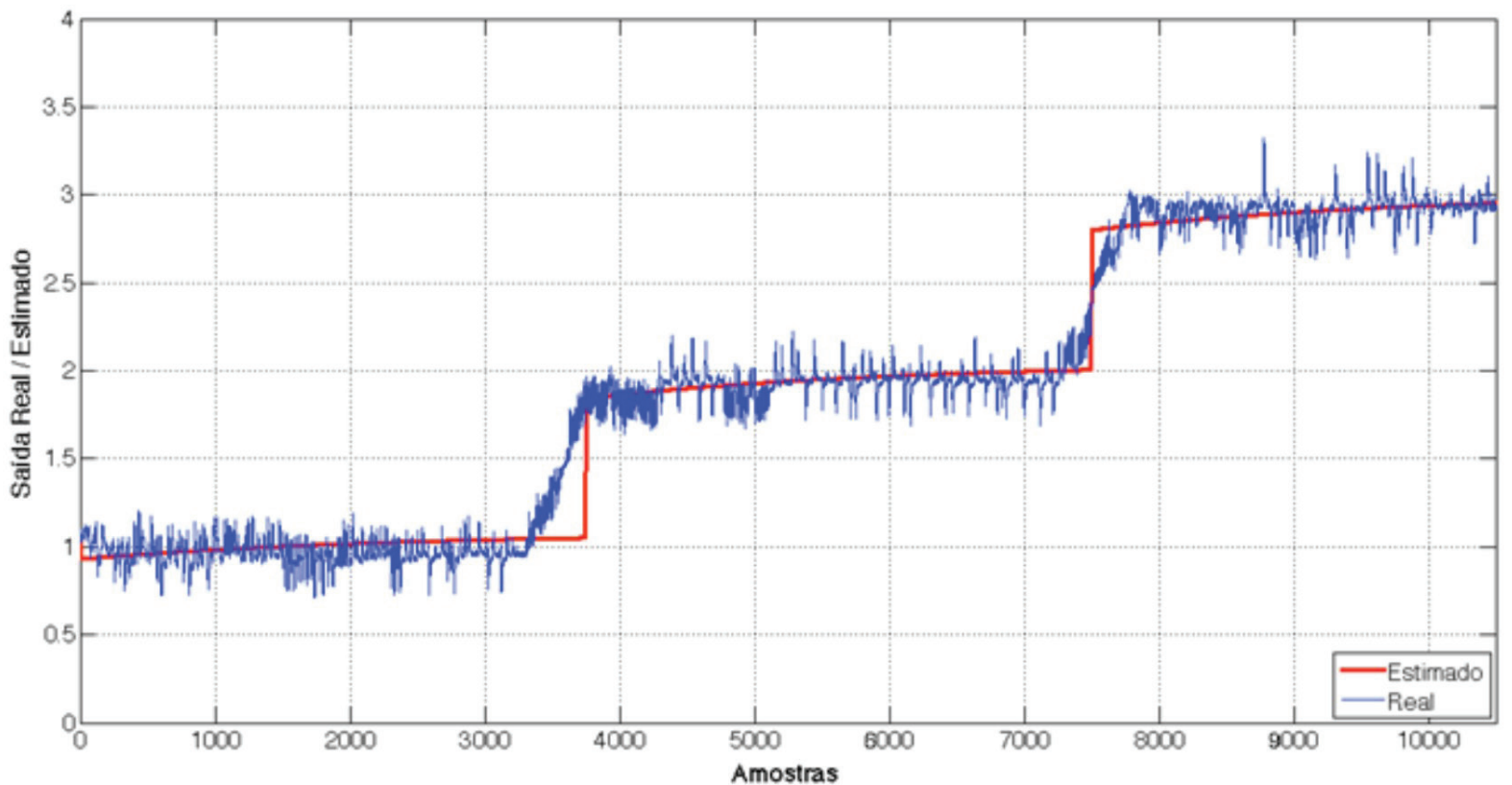


Os resultados obtidos de identificação são os seguintes (Tabela 2):

Tabela 2: Resultados da identificação.

\begin{tabular}{cc}
\hline Índice & Valor \\
\hline FPE & 0.01658 \\
Função Custo & 0.01657 \\
Fit (\%) & 83,2 \\
\hline
\end{tabular}

Em que:

- FPE (Final Predictive Error): Fornece uma medida da qualidade do modelo estimado, onde, quanto mais preciso, menor seu FPE.

- Função Custo: Indica o somatório do quadrado das diferenças entre o modelo estimado e os dados coletados. Quanto menor a diferença, menor é seu valor.

- Fit (\%): É uma medida percentual que indica quanto o modelo estimado é fiel aos dados coletados. Quanto mais fiel, maior é seu fit.

Utilizando o restante das amostras coletadas para validação do modelo estimado, tem-se como resultado a Figura 10.

Figura 10: Validação do modelo estimado.

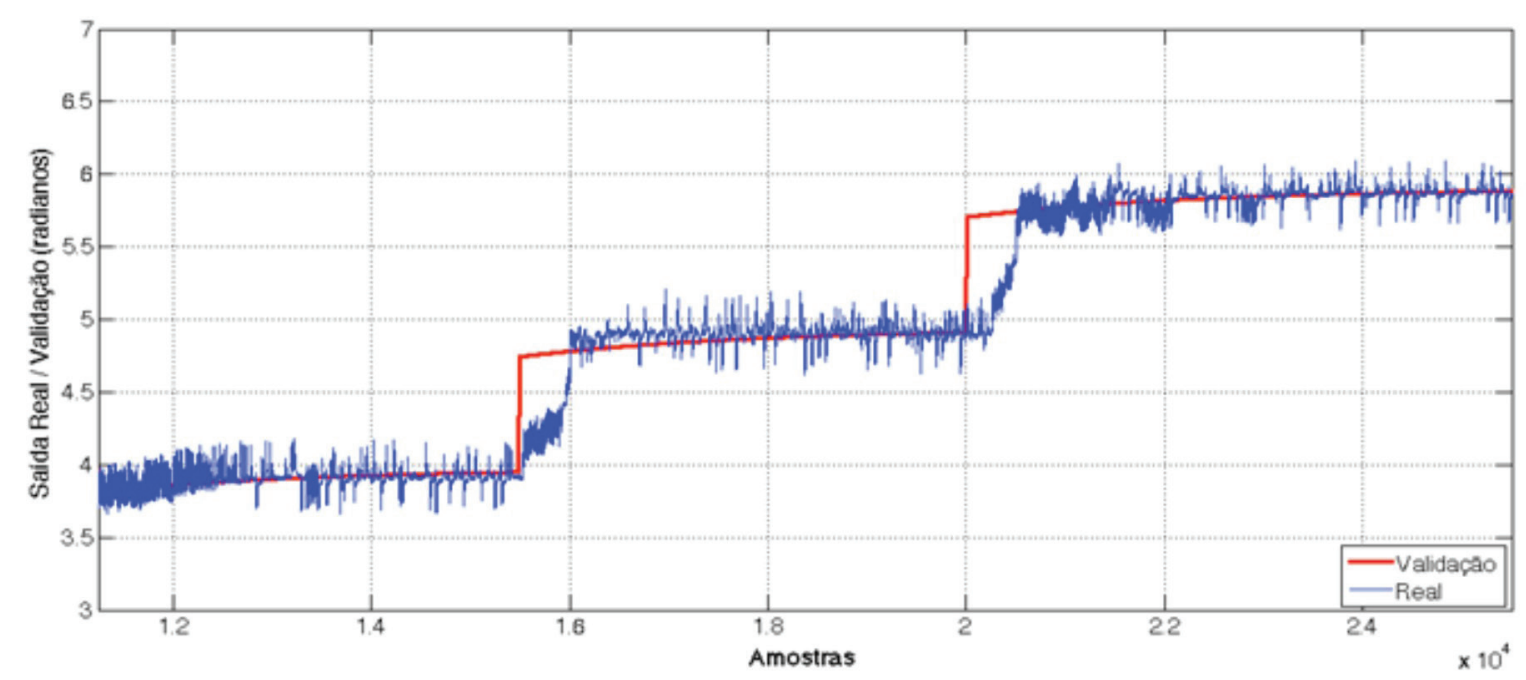

Os resultados obtidos de validação constam na Tabela 3.

Tabela 3: Resultados da validação.

\begin{tabular}{cc}
\hline Índice & Valor \\
\hline FPE & 0.01658 \\
Função Custo & 0.01657 \\
Fit (\%) & 77,23 \\
\hline
\end{tabular}

\section{Conclusão}

Com a utilização da técnica não linear de Hammerstein, foi possível representar as dinâmicas de um motor de indução trifásico do tipo gaiola de esquilo.

Com os resultados adquiridos, tal como os índices de validação, é correto afirmar que o uso da técnica não linear de Hammerstein representa as características do processo em questão. Já que, mesmo com a utilização de uma realimentação de baixo custo, como o potenciômetro, o que insere muitos ruídos no sistema, foi possível se chegar a resultados excelentes. 
Além do mais, a técnica utilizada também representou a variação dos parâmetros do sistema, tal como a resistência rotórica, que varia com a temperatura. Como foram realizadas diversas amostras, a temperatura do sistema variou consideravelmente e, consequentemente, a resistência rotórica. Mesmo assim, os resultados adquiridos foram bons.

Com o resultado deste trabalho, propõe-se a utilização de estratégias de controle não linear, tal como GPC (Generalized Predictive Control), com a utilização do modelo não linear de Hammerstein.

Como realizado em Silva et al. (2015) - uma estratégia de controle GPC com o modelo linear CARIMA (Controlled Auto-Regressive and Integrated Moving Avarage) -, propõe-se o mesmo estudo, com a substituição do modelo linear pelo não linear discutido neste trabalho.

Já Souza Júnior et al. (2013) utilizam uma estratégia de controle híbrida, em que, através de lógica fuzzy, o controlador se comporta tanto como um PID (Proporcional-Integral-Derivativo) como um GPC, a fim de se conseguir um melhor desempenho. Também é utilizado um modelo CARIMA, o qual se propõe a utilizar as mesmas técnicas de controle com o uso do modelo não linear de Hammerstein obtido neste trabalho.

\section{Referências}

AGUIRRE, L. A. Introdução à identificação de sistemas: técnicas lineares e não lineares aplicadas a sistemas reais. 3. ed. Belo Horizonte: UFMG, 2007.

AL-DUWAISH, H.; KARIM, M. N. A new method for the identification of Hammerstein model. Automatica, Torino, v. 33, n. 10, p. 1871-1875, oct. 1997.

BOSE, B. K. Power electronics and AC drivers. New Jersey: Prentice-Hall, 1986.

BOUTAYEB, M.; AUBRY, D.; DAROUACH, M. A robust and recursive identification method for the Hammerstein model. In: IFAC TRIENNIAL WORLD CONGRESS, 13., 1996, San Francisco. Anais... San Francisco: Pergamon Press, 1996. p. 447-452.

COELHO, A. A. R.; COELHO, L. S. Identificação de sistemas dinâmicos lineares. Florianópolis: UFSC, 2004.

COELHO, A. A. R.; SANTOS, J. E. S. Identificação e controle preditivo para o modelo de Hammerstein: abordagem não paramétrica. In: CONGRESSO BRASILEIRO DE AUTOMÁTICA, 15., 2002, Natal. Anais... Natal: Sociedade Brasileira de Automática, 2002. p. 2810-2815.

DING, F.; LIU, X. P.; LIU, G. Identification methods for Hammerstein nonlinear systems. Digital Signal Processing, Pisa, v. 21, n. 2, p. 215-238, mar. 2011.

DINIZ, E. C.; SOUZA JÚNIOR, A. B.; HONÓRIO, D. A. Análise comparativa entre os algoritmos de controle vetorial e de modo de controle deslizante para o controle de posição de um motor de indução trifásico. Revista Tecnologia, Fortaleza, v. 31, n.2, p. 217-229, dez. 2010.

ESKINAT, E.; JOHSON, S. H.; LUYBEN, W. L. Use of Hammerstein models in identification of nonlinear systems. AlChE Journal, New York, v. 37, n. 2, p. 255-268, feb. 1991.

FRUZZETI, K. P.; PALAZOGLU, A.; MCDONALD, K. A. Nonlinear method predictive control using Hammerstein models. Journal of Process Control, Louvain-la-Neuve, v. 7, n.1, p. 31-41, feb. 1997.

HASEGAWA, M.; OGAWA, D.; MATSUI, K. Parameter identification scheme for induction motors using output intersampling approach. Asian Power Electronics Journal, Kowloon, v. 2, n.1, p. 15-22, apr. 2008.

LJUNG, L. System identification: theory for the user. New Jersey: Prentice-Hall, 1999.

LJUNG, L., SCHOUKENS, J., SUYKENS, J. Wiener-Hammerstein benchmark. In: IFAC SYMPOSIUM ON SYSTEM IDENTIFICATION, 15., 2009, Saint-Malo. Anais... Saint-Malo: International Federation of Automatic Control, 2009. p. 1074-1079.

NARENDRA, K. S.; GALLMAN, P. G. An iterative method for the identification of nonlinear systems using Hammerstein model. IEE Transactions on Automatic Control, Notre Dame, v. 11, n. 3, p. 546-550, jul. 1966.

SANTOS, J.E.S. Controle preditivo não linear para sistemas de Hammerstein. 2007. 139p. Tese (Doutorado em Engenharia Elétrica) - Programa de Pós-Graduação em Engenharia Elétrica, Universidade Federal de Santa Catarina, Florianópolis, 2007. 
SILVA, W. A. et al. Generalized predictive control robust for position control of induction motor using field-oriented control. Electrical Engineering, Aachen, v. 97, n. 3, p. 195-204, jan. 2015.

SOUZA JÚNIOR, A. B. S et al. Generalized predictive control applied to the position control of a induction motor. In: IEEE/IAS INTERNATIONAL CONFERENCE ON INDUSTRY APPLICATIONS, 10., 2012, Fortaleza, Anais... Fortaleza: IEEE Industry Applications Society, 2012. p. 1-6.

SOUZA JÚNIOR, A. B.; et al. Hybrid position control technique of induction motor drive. Control and Cybernetics, Warzawa, v. 42, n. 4, p. 754-772, oct. 2013.

ZHU, X. E SEBORG, D.E. Nonlinear predictive control based on Hammerstein models. In: INTERNATIONAL SYMPOSIUM ON PROCESS SYSTEMS ENGINEERING (PSE'94), 5, 1994, Kyongju Korea, Anais... Kyongju, Korea: PSE'94, 1994. p. 995-100.

Sobre os autores

\section{Lucas Rabelo Rebouças}

Engenheiro de Controle e Automação, Universidade de Fortaleza - UNIFOR. Mestrando do Departamento de Engenharia Elétrica da Universidade Federal do Ceará - UFC.

\section{Antônio Barbosa de Souza Júnior}

Engenheiro de Controle e Automação, Universidade de Fortaleza - UNIFOR. Mestre e Doutor em Engenharia Elétrica pela Universidade de Federal do Ceará - UFC. Professor do Instituto Federal de Educação, Ciências e Tecnologia - IFCE.

\section{Tobias Rafael Fernandes Neto}

Engenheiro Eletricista, Universidade de Fortaleza - UNIFOR. Mestre em Engenharia Elétrica pela Universidade Federal do Ceará - UFC. Doutor em Engenharia Elétrica pela Technische Universität Darmstadt - TUD, Alemanha. Professor adjunto do Departamento de Engenharia Elétrica da Universidade Federal do Ceará - UFC.

\section{Laurinda Lúcia Nogueira dos Reis}

Engenheira Eletricista, Universidade Federal do Ceará - UFC. Mestre em Engenharia Elétrica pela Universidade Federal da Paraíba - UFPB. Doutora em Engenharia Elétrica pela Universidade Federal de Santa Catarina - UFSC. Professora adjunta do Departamento de Engenharia Elétrica da Universidade Federal do Ceará - UFC.

\section{Luiz Henrique Silva Colado Barreto}

Engenheiro Eletricista, Universidade Federal de Mato Grosso - UFMT. Mestre e Doutor pela Universidade Federal de Uberlândia - UFU. Professor adjunto do Departamento de Engenharia Elétrica da Universidade Federal do Ceará - UFC. 